\title{
Serial siphon valving for centrifugal microfluidic platforms
}

\author{
Jonathan Siegrist $\cdot$ Robert Gorkin $\cdot$ Liviu Clime $\cdot$ \\ Emmanuel Roy $\cdot$ Régis Peytavi $\cdot$ Horacio Kido $\cdot$ \\ Michel Bergeron · Teodor Veres • Marc Madou
}

Received: 17 September 2009/Accepted: 1 October 2009/Published online: 4 November 2009

(C) The Author(s) 2009. This article is published with open access at Springerlink.com

\begin{abstract}
Today, the focus in microfluidic platforms for diagnostics is on the integration of several analysis steps toward sample-to-answer systems. One of the main challenges to integration is the requirement for serial valving to allow the sequential release of fluids in a temporally and spatially controlled manner. The advantages offered by centrifugal microfluidic platforms make them excellent candidates for integration of biological analysis steps, yet they are limited by the lack of robust serial valving technologies. This is especially true for the majority of centrifugal microfluidic devices that rely on hydrophilic surfaces, where few passive serial valving techniques function reliably. Building on the useful functionality of centrifugal microfluidic siphoning previously shown, a novel serial siphon valve is introduced that relies on multiple, inline siphons to provide for a better controlled, sequential release of fluids. The introduction of this novel concept is followed by an analytical analysis of the device.
\end{abstract}

Electronic supplementary material The online version of this article (doi:10.1007/s10404-009-0523-5) contains supplementary material, which is available to authorized users.

J. Siegrist $(\square) \cdot$ R. Gorkin $\cdot$ M. Madou

Department of Biomedical Engineering, University

of California, Irvine, Irvine, CA 92697, USA

e-mail: jsiegris@uci.edu

L. Clime $\cdot$ E. Roy $\cdot$ T. Veres

National Research Council - Industrial Materials Institute,

Boucherville J4B 6Y4, Canada

R. Peytavi · M. Bergeron

University of Laval, Quebec City, QC G1V4G2, Canada

H. Kido $\cdot$ M. Madou

Department of Mechanical and Aerospace Engineering,

University of California, Irvine, Irvine, CA 92697, USA
Proof-of-concept is also demonstrated, and examples are provided to illustrate the range of functionality of the serial siphon valve. The serial siphon is shown to be robust and reproducible, with variability caused by the dependence on contact angle, rotation velocity, and fluidic properties (viz., surface tension) significantly reduced compared to current microfluidic, centrifugal serial valving technologies.

Keywords Centrifugal $\cdot$ Microfluidic $\cdot$ Siphon $\cdot$ Valve

\section{Introduction}

As the application of microfluidics for biological analysis, and in particular nucleic acid (NA) diagnostics, becomes more widespread, emphasis is being placed on adoption of standardized platforms. Centrifugal microfluidics is one such platform with many advantages over typical, pressure-pump driven systems (Madou 2002; Madou et al. 2006). For example, centrifugal pumping eliminates the need for large power supplies and/or pumps to drive fluids (only a low-power motor is needed), and pumping is not strongly dependent on the $\mathrm{pH}$ or ionic strength of the fluid (Madou et al. 2006). Compact-disc (CD) like centrifugal fluidic platforms have been the focus of many R\&D efforts for almost 40 years (Madou et al. 2006), and continue to be of interest in both academia and industry as integration of multiple biological analysis steps and functions becomes a focus (Beebe et al. 2002; Cho et al. 2007b; Ducrée et al. 2007; Duffy et al. 1999; Haeberle and Zengerle 2007; Madou et al. 2006).

1.1 Valving on centrifugal microfluidic platforms

As with any fluidic platform, the fundamental problem of moving liquids to and from different areas of interest in a 
controlled manner can be simplified down to the need for two technologies: pumps and valves. Pumping on centrifugal platforms is most commonly performed by centrifugally induced flow, dependent in part on rotation frequency, radial distance from the CD center, and channel geometry. Valving is typically passive, and takes on the form of either hydrophobic or capillary valves, which depend on surface tension forces (Madou et al. 2006). Hydrophobic valves can take on two different forms: one utilizing changes in channel geometries and the other utilizing surface modification. In both cases, the fluid can be forced past the hydrophobic valve by increasing the spin frequency beyond a critical burst value.

The capillary valve is more commonly used in microfluidic CD platforms, and is a result of the balance between centrifugal and surface tension forces in a hydrophilic material (Cho et al. 2007a). In a CD with hydrophilic surfaces, fluids are passively pumped through microchannels due to capillary forces. During spinning of the disc, centrifugal forces on the fluids can aid this movement. As fluids reach an abrupt widening, a larger surface tension force develops. If the surface tension force is greater than that of the centrifugal force, then the fluid flow will stop even though the CD continues to spin. At a certain spin speed, known as the burst frequency, the centrifugal force will overcome the surface tension force and the fluid will continue down the channel. By designing microfluidic structures with channels of varying capillary sizes, control of when a valve "opens" can be achieved simply by increasing the rotational speed of the CD.

Another type of passive valve used on CD microfluidic platforms is the siphon valve (Ducrée et al. 2007; Madou et al. 2006). A common implementation of the siphon valve relies on a hydrophilic environment, such that the siphon channel is primed using capillary forces only (Kido et al. 2007). A typical siphon valve system consists of a liquid-filled chamber/reservoir with a connected siphon channel first extending upwards above the radial position of the chamber and then down to a position radially below the chamber. During high-speed rotation, centrifugal forces keep the liquid retained inside the chamber and inside the siphon channel (Fig. 1a, left). By reducing the rotation speed, a critical spin speed is reached where the centrifugal forces are no longer dominant over the capillary forces inside the siphon channel. At this point, capillary forces pump fluid over the siphon channel crest, and down the siphon channel to the entrance of a radially lower chamber (which itself serves as a capillary valve, as discussed above). At this point, the siphon channel fluid front (meniscus) is at a point radially below the bulk liquid inside the upper chamber, and the siphon is now primed (Fig. 1b, left). Once the CD rotation velocity is increased, fluid is completely pumped out of the upper chamber and

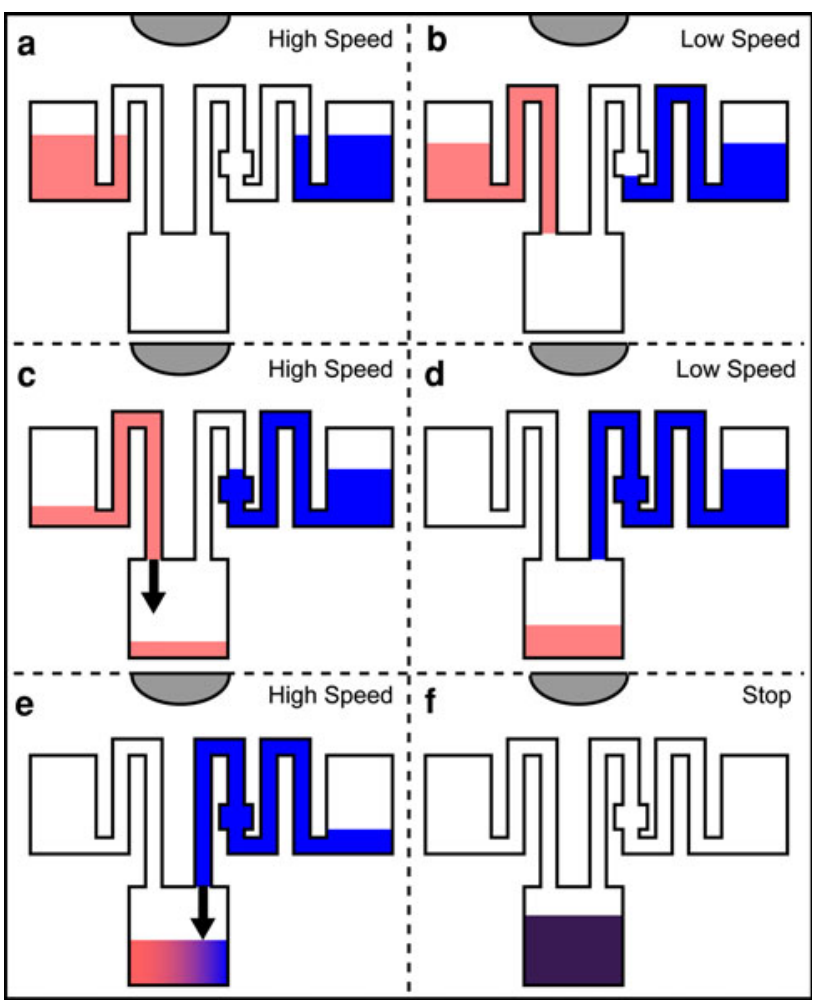

Fig. 1 Schematic showing the simultaneous function of single (left, red) and double serial (right, blue) siphon valve channels between chambers. The serial siphon valve structure contains a capillary valve inline with the second siphon; the $\mathrm{CD}$ center is above each figure. a Liquids are loaded in each reservoir and the CD spin speed increased to high. $\mathbf{b}$ Spin speed is decreased to low, allowing the single siphon to prime, and the double siphon to prime up to, but not past, the inline capillary valve. $\mathbf{c}$ High spin speed begins siphon pumping of the single siphon, and allows the double siphon to overcome the inline capillary valve. d Spin speed is decreased to low, allowing the double siphon to finish priming. e High spin speed begins siphon pumping of the double siphon. f The CD is stopped, with single and double siphoning having sequentially moved liquid into the lower chamber

into the lower chamber through siphoning (Fig. 1c, left). Figure 1a-d shows the function of a single siphon (left).

Microfluidic siphons are extremely useful on CD platforms where they can serve several functions, often time in combination, such as valving, pumping, and liquid metering and volume definition (Cho et al. 2007a; Ducrée et al. 2007; Steigert et al. 2007). The main drawback of siphoning on the $\mathrm{CD}$ is the requirement of a hydrophilic channel, which is most often obtained by surface treatment of an originally hydrophobic surface. Surface treatments add complexity to characterization of fluidic behavior, as they can show a dynamic behavior over time as surface energy decreases (Beaulieu et al. 2009; Larsson and Dérand 2002). For example, polycarbonate can be exposed to oxygen-plasma to create a hydrophilic surface. However, this surface treatment methodology is time dependent, and treatments can degrade over a time scale of days (Larsson and Dérand 2002). 


\subsection{Serial valving on centrifugal microfluidic platforms}

The integration of various analysis steps continues to be a focus for microfluidic diagnostic devices, and the need for serial, or sequential, valves is of extreme importance; various liquids with different properties (e.g., surface tension and viscosity) must be released at the appropriate time and to the appropriate location. Serial capillary valves in centrifugal microfluidic platforms have been demonstrated, most commonly in hydrophobic environments, with considerable success (Badr et al. 2002; Jia et al. 2006; Lai et al. 2004; Madou et al. 2006; Peytavi et al. 2005). In hydrophilic environments, however, capillary valves are more difficult to control and characterize (viz., determination of burst frequency) and are more prone to change over time. The burst frequency is largely dependent on the surface energy of the material, which can vary depending on the surface treatment conditions used and the dynamic time dependency of the contact angle. For example, oxygen-plasma-treated polycarbonate can undergo surface treatment degradation $<30$ days after treatment (Larsson and Dérand 2002). It remains difficult to robustly characterize capillary valve burst frequencies to define operation parameters when using hydrophilic materials. In addition, spin profiles are limited by capillary valve burst frequencies, preventing high-speed spin operations (e.g., centrifugation). To make the situation more difficult, there are few other, if any, passive, sequential valving methods available for hydrophilic centrifugal platforms.

Thus, there remains a need for a robust serial valve on microfluidic centrifugal platforms that can be implemented in a passive manner using a hydrophilic material, especially when the material is made hydrophilic through the use of surface treatments. Here, a novel valve that meets these requirements is presented that relies on the movement of fluid through a serial progression of siphons. The valve is robust, characterizable, and its operation is not highly dependent on variations or changes in contact angles, fluidic properties, or rotation velocity fluctuations.

\section{Serial siphon fundamentals}

The serial siphon consists of a sequence of multiple siphon features, with capillary valves imbedded inline between each siphon (Bergeron et al. 2008; Roy et al. 2008). Note that siphon valving requires a hydrophilic material in the siphon channels, to allow capillary pumping. Fig. 1 shows the simplest example implemented with a double siphon device (right). After introduction of the fluid to the upper chamber, the $\mathrm{CD}$ rotation velocity is increased to a high speed such that the fluid is trapped within the siphon channel and liquid chamber (Fig. 1a, right). Once the CD speed is reduced to allow capillary force to overcome centrifugal force, the siphon liquid primes up and over the first siphon crest and to the first inline capillary valve (Fig. 1b, right).

The abrupt widening at the inline capillary valve creates an area where the surface tension forces dominate the capillary and centrifugal pumping forces, and capillary pumping (i.e., siphon priming) ceases. In order to burst the inline capillary valve, the CD spin speed is then increased to a higher value, which results in the centrifugal forces overpowering the inline capillary valve surface tension forces. The siphon liquid progresses up the next siphon to a position radially equivalent to the chamber liquid height (Fig. 1c, right). When the $\mathrm{CD}$ rotation speed is again lowered, capillary pumping primes the liquid over the crest of the second siphon loop and down to the lower chamber, to a height radially below that of the upper chamber liquid (Fig. 1d, right). When the CD speed is increased again, the liquid pumps completely out of the chamber, through both siphon loops and the inline capillary valve, and into the lower chamber (Fig. 1e, right). In this way, the liquid is serially controlled through 2 "digital" spin speed cycleshigh (1), low (0), high (1), low (0), and high (1).

The functionality of this device can be extrapolated to include triple and higher multiple serial siphons. Moreover, true serial valving functionality is obtained, for example, by putting a single loop on one chamber and a double loop on a second chamber (Fig. 1). In this way, fluid is released from each chamber sequentially during each high-low cycle of CD spin speed control. This serial siphon concept provides powerful passive valving functionality on hydrophilic centrifugal microfluidic platforms.

\section{Materials and methods}

\subsection{CD fabrication}

The CD devices tested here consist of multi-layer structures made of inexpensive polycarbonate plastic and double-sided, pressure-sensitive adhesives (PSA). Using a relatively simple computer-numerical control (CNC) machine (T-Tech, GA, USA-QuickCircuit 5000), channel widths down to $1 \mathrm{~mm}$ can be machined into stock polycarbonate plastic (McMaster-Carr, CA, USA). A cutterplotter (Graphtec, Japan-Graphtec CE-2000) can be used to cut channel widths as narrow as $200 \mu \mathrm{m}$ in thinner materials such as $100 \mu \mathrm{m}$-thick PSA (FLEXcon, MA, USADFM 200 Clear V-95 150 POLY H-9 V-95 4). Once the appropriate pieces have been designed and machined, they are aligned centrally and radially and laminated together using the PSA layers.

The microfluidic CD devices presented here consist of five layers: (1) top polycarbonate $\mathrm{CD}(\sim 1 \mathrm{~mm}$ thick) with 


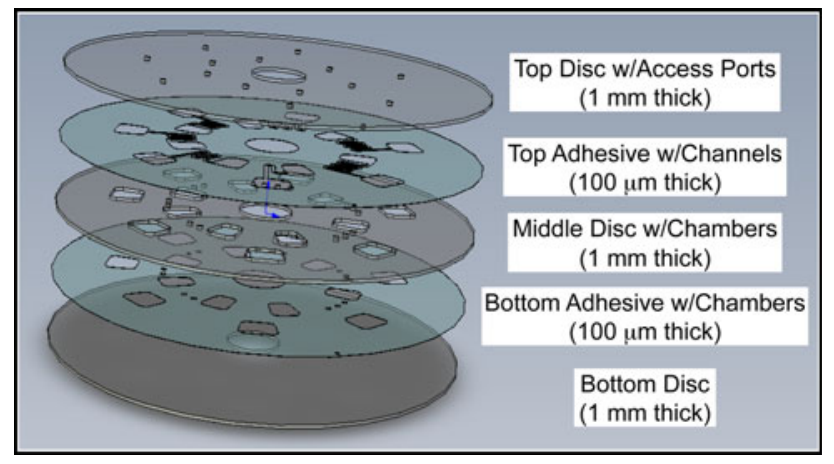

Fig. 2 Schematic showing assembly of the microfluidic CD, consisting of polycarbonate and pressure-sensitive adhesive layers

CNC-machined sample loading and air venting holes, (2) pressure-sensitive adhesive with siphon microchannel features cut using a plotter, (3) middle polycarbonate CD ( $\sim 1 \mathrm{~mm}$ thick) with CNC chamber features, (4) pressuresensitive adhesive cut using a plotter, and (5) solid bottom polycarbonate $\mathrm{CD}$ ( $\sim 1 \mathrm{~mm}$ thick) to seal off the channels (Fig. 2).

The $\mathrm{CD}$ devices tested rely on $\mathrm{O}_{2}$ plasma treatment to render the polycarbonate surface hydrophilic, essential to function of the siphons. A Technics 500II Asher was used at an $\mathrm{O}_{2}$ pressure of $200 \mathrm{mTorr}$ with $200 \mathrm{~W}$ of power for $2.5 \mathrm{~min}$ for hydrophilization of the unassembled polycarbonate $\mathrm{CD}$ layers. The $\mathrm{CD}$ devices were tested within 7 days of $\mathrm{O}_{2}$ plasma treatment.

\subsection{Serial siphon design}

The CD designed for proof-of-concept consists of simple serial siphon devices, in which two chambers are connected with various implementations of the serial siphon, including single, double, and triple siphons. For simplicity, set geometries for only the triple siphon are focused on here for testing and analysis as a model for both simpler serial siphons (double) and more complex serial siphons (e.g., quadruple) of varying dimensions.

The serial siphon devices consist of $1.2 \mathrm{~mm}$ deep chambers capable of holding over $40 \mu \mathrm{l}$ of solution, connected by serial siphon channels $100 \mu \mathrm{m}$ deep and $1 \mathrm{~mm}$ wide. Spacing between the serial siphons is $1 \mathrm{~mm}$, and circular, inline capillary valves $1.2 \mathrm{~mm}$ deep with a diameter of $1.5 \mathrm{~mm}$ are used (Fig. 3). The distance from the CD center $(R)$ of the serial siphon crests is 22.0, 22.4, and $23.5 \mathrm{~mm}$ for each of $\mathrm{C}_{1}, \mathrm{C}_{2}$, and $\mathrm{C}_{3}$, respectively, representing an average siphon crest $R$ of $22.6 \mathrm{~mm}$ (Fig. 3).

\subsection{Experimental setup}

Microfluidic testing of the serial siphon CDs was carried out by strobe-imaging of the CDs during operation. $40 \mu \mathrm{l}$

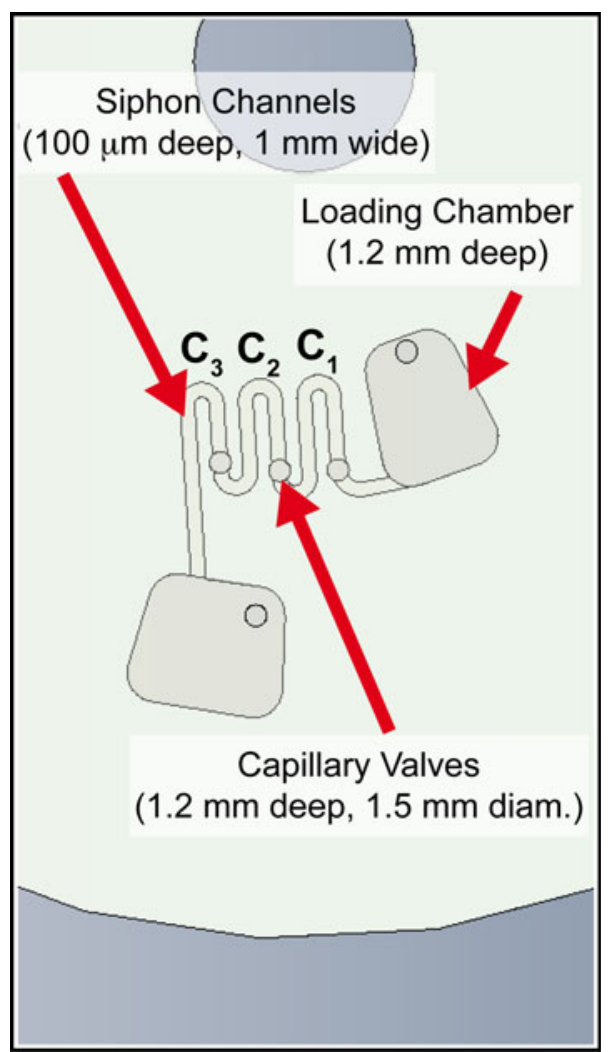

Fig. 3 Schematic showing the triple siphon valve device designed, tested, and analyzed; each siphon crest is labeled. The first inline capillary valve prevents undesired siphon priming during liquid loading. The remaining inline capillary valves ensure only one siphon primes at a time as the spin speed is cycled between high and low values

of DI water containing $<1 \% \mathrm{v} / \mathrm{v}$ of a contrast agent (McCormick, MD, US-Neon food dye) was placed inside the $\mathrm{CD}$ reservoir, and the $\mathrm{CD}$ placed on a spin-stand equipped with a rotational motor (Pacific Scientific Servo Motor) connected to an amplifier/controller (PAC SCI Programmable Servo Drive), which enables various rotational profiles to be run. The CDs to be tested were placed on an aluminum chuck coupled to the motor shaft and locked in place. The servo drive uses an interface program, ToolPAC, in order to easily configure and program the motor for specific applications. For testing of serial siphon functionality, CDs were subjected to cyclic spin profiles at speeds of 600 revolutions-per-minute (RPM) and 1,000 RPM, with an acceleration and deceleration of $500 \mathrm{RPM} / \mathrm{s}$. For determination of the critical siphon priming frequency, the CDs were spun at a speed of 1,000 RPM, and then slowed down in intervals of 10 RPM. The spin speed at which each of the siphons primed was recorded. The observed experimental siphon priming frequency was $770 \pm 40$ RPM (1 std. deviation) among six device replicates on two different CDs. 
An imaging system was utilized that allows viewing of a sequence of color images of the area of interest on the CD in real time (while it is rotating) and storage of the captured frames on a computer. The imaging system is composed of: a camera (Basler A301bc, $640 \times 480$ pixels, 80 fps max., and $10 \times$ zoom lens mounted), a strobe light (PerkinElmer MVS-4200, $6 \mu$ s duration), and a retro-reflective fiberoptic sensor (Banner D10 Expert Fiber-Optic Sensor). The strobe light, with a $100 \mathrm{~Hz}$ maximum repetition frequency, is employed to reduce blurry images of the fast moving CD. In order to generate synchronized signals, a reflective marker $(\sim 2 \mathrm{~mm} \times 2 \mathrm{~mm})$ is placed on the surface of the $\mathrm{CD}$ and aligned with the fiber-optic sensor. When the sensor detects the marker, a signal pulse is sent to the video capture board, triggering the camera and strobe light to acquire one image frame per $\mathrm{CD}$ revolution.

After image acquisition, movie frames were extracted showing the height of the liquid in each siphon channel at a steady-state spin speed (Fig. 4). Vector-based drawing software was then used to measure the distance of the meniscus in the serial siphons and the reservoir from the CD center, through measurement estimation by pixelization (conversion to bitmap) of raw images captured from the video. These measurements were used in the analysis to follow.

\section{Analytical analysis}

Here, a practical analysis of the serial siphon is performed to provide guidance for design of serial siphon devices. The most crucial aspect of any centrifugal microfluidic siphon, serial or otherwise, is the specific priming spin frequency value. Above this spin speed, the siphon liquid will be prevented from priming over the siphon crest, and below this value priming over the crest occurs. With an analytical model, one can design serial siphon structures to have siphon burst frequencies within a desired range.

Another crucial component of the serial siphon is the inline capillary valve. As discussed above, capillary valving on centrifugal microfluidic platforms has been studied extensively, and so it will not be addressed in detail here (Chen et al. 2008; Madou et al. 2006). The burst frequency of a capillary valve can be controlled by the valve geometry. The only true requirement of the capillary valve(s) for the serial siphon structures is that the burst frequency be above the lower limit of rotation speed for the control system. In theory, this is 0 RPM, and so the capillary valve requirements are minimal.

Analysis of the serial siphon consists, fundamentally, of a force balance (more precisely a pressure balance) between the centrifugal pumping force, acting in a direction radially outward from the CD center, and the capillary priming force, acting in a direction along the siphon channel and chamber toward the CD center. The relevant RPM burst frequency value for siphon priming is of interest here, and so the system modeled is taken to be in a steady-state mode. Dynamic forces, such as the Coriolis force, are not present in such equilibrium states, and so are not considered (Brenner et al. 2005). The channels being modeled have a dimension in the $\mathrm{Z}$-axis (out of the $\mathrm{CD}$ plane) of $100 \mu \mathrm{m}$, which is much smaller than the $\mathrm{X}-\mathrm{Y}$ dimension of the channel, $1 \mathrm{~mm}$. Thus, a 2-D model is utilized. Water containing $<1 \%$ contrast agent was used as the test fluid, and so DI water is taken as the sample for use in analysis and calculations.

First, a reservoir and a radial capillary microchannel connecting the reservoir to a vent near the $\mathrm{CD}$ rotation center are considered (Fig. 5a). As the CD spins, the condition of liquid flow-stop in the microfluidic capillary


Fig. 4 Time-lapse sequence of photos showing function of the triple serial siphon valve as it undergoes a cyclic sequence of high and low spin speeds; the thin white bar demarcates the approximate meniscus

locations. See Movie A for a complete video of the triple serial siphon valve sequence 
channel (i.e., the centrifugal and capillary pressure balance) is given as:

$\frac{1}{2} \rho \omega^{2}\left(R_{0}-R\right)\left(R_{0}+R\right)=\left(\frac{\gamma}{H}-\frac{\gamma}{H_{\text {res }}}\right)\left(2 \cos \theta_{\mathrm{C}}\right)$

where $R_{0}$ and $R$ stand, respectively, for the positions of the liquid in the reservoir and the microfluidic channel, $\rho$ is the density of water $\left(1,000 \mathrm{~kg} / \mathrm{m}^{3}\right)$, and the $\gamma$ surface tension coefficient $\left(0.0728 \mathrm{~N} / \mathrm{m}\right.$ ) (Madou 2002). $H$ and $H_{\text {res }}$ are the depths of the channel $(100 \mu \mathrm{m})$ and reservoir $(1.2 \mathrm{~mm})$, respectively, and $\theta_{\mathrm{C}}$ represents the contact angle on the floor and ceiling (for both the microfluidic channel and reservoir). In the case presented, both the floor and ceiling consist of the same oxygen-plasma-treated polycarbonate, and so they have the same $\theta_{\mathrm{C}}$ value; $\omega=2 \pi f$ is the angular velocity, and $f$ the frequency.

Considering the notation:

$\Gamma=\frac{2 \gamma}{H}-\frac{2 \gamma}{H_{\mathrm{res}}}$

then Eq. 1 above can be rewritten as:

$\rho \omega^{2}\left(R_{0}^{2}-R^{2}\right)=2 \Gamma \cos \theta_{\mathrm{C}}$

From here, dependence of the position of the liquid meniscus in the capillary siphon channel, $R$, on the CD spin frequency, $f$, can be solved from Eq. 2 as:

$R(f)=\sqrt{R_{0}^{2}-\frac{\Gamma}{2 \pi^{2} \rho f^{2}} \cos \theta_{\mathrm{C}}}$

A qualitative plot of this function is shown in Fig. 5a.
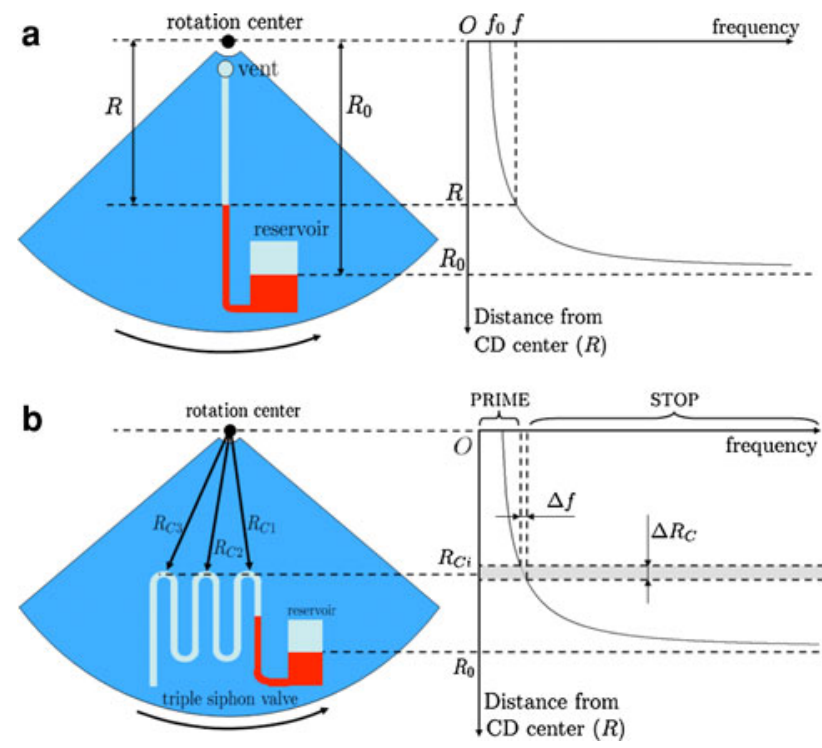

Fig. 5 a Schematic drawing of the capillary flow in a perfectly radial microfluidic channel. This was used in order to deduce $R(f)$. b The actual triple siphon valve
It can be observed from Eq. 3 that $R=R_{0}$ as $f \rightarrow \infty$. In other words, at a very high spin frequency, the height of the liquid in the siphon channel will be practically equal to that of the liquid height in the reservoir. The condition $R=0$, that is the meniscus in the capillary microchannel reaches the $\mathrm{CD}$ rotation center, is fulfilled for:

$f_{0}=\frac{1}{2 \pi R_{0}} \sqrt{\frac{2 \Gamma \cos \theta_{\mathrm{C}}}{\rho}}$

Note that $R_{0}$ (the level of liquid in the reservoir) is considered a constant parameter here, as the decrease is negligible during serial siphon priming (Fig. 4). In reality, as the serial siphons prime, $R_{0}$ increases, and the calibration curve, as shown in Fig. 5a, changes slightly.

In order to have the liquid meniscus stopped at a frequency of rotation $f_{\text {stop }}$ in the capillary channel (Fig. 5b) for a given siphon with a crest at a position $R_{\mathrm{c}}$ from the CD center, the condition:

$R\left(f_{\text {stop }}\right)>R_{\mathrm{c}}$

must be fulfilled. The liquid will consequently prime (overtake the crest) if:

$R\left(f_{\text {prime }}\right) \leq R_{\mathrm{c}}$

Thus, there exists a critical siphon priming frequency at which the capillary force will allow priming all of the way up to the siphon crest. At this point, the liquid will continue to wick over and down the next siphon channel, completing siphon priming. In the triple siphon presented here, the crests are not all at exactly the same distance from the CD center, and so a certain interval, $\Delta R_{\mathrm{c}}$, will separate the regions of priming and stop of the valve (Fig. 5b). Ideally, the interval $\Delta R_{\mathrm{c}}=\max \left\{R_{\mathrm{Ci}}\right\}-\min \left\{R_{\mathrm{Ci}}\right\}$ should be minimized; that is, the crests should be at approximately the same distance from the CD center. If this is not the case, there will be an interval of priming frequencies, $\Delta f$, spanning the siphon structure.

In the analytical siphon analysis performed here, the triple serial siphon is considered as described in the Serial siphon design Sect. 3.2, above. Fluidic properties ( $\rho$ and $\gamma$ ) were taken for water, as discussed above. The remaining unknown, $\theta_{\mathrm{C}}$, was calculated based on the experimental images collected, as follows. Stop flow condition for a single siphon was imaged at 1,000 RPM spin speed, and the respective $R_{0}$ and $R$ values were measured, as discussed above, to be 26.7 and $24.3 \mathrm{~mm}$. Solving Eq. 3 for $\theta_{\mathrm{C}}$ gave an average contact angle of $59^{\circ}$. This value correlates with contact angles of DI water on oxygen-plasma-treated polycarbonate surfaces found in the literature, and was used for the remaining analysis (Larsson and Dérand 2002). With all variables defined, Eq. 3 was plotted for the given triple siphon geometry and conditions (Fig. 6). 


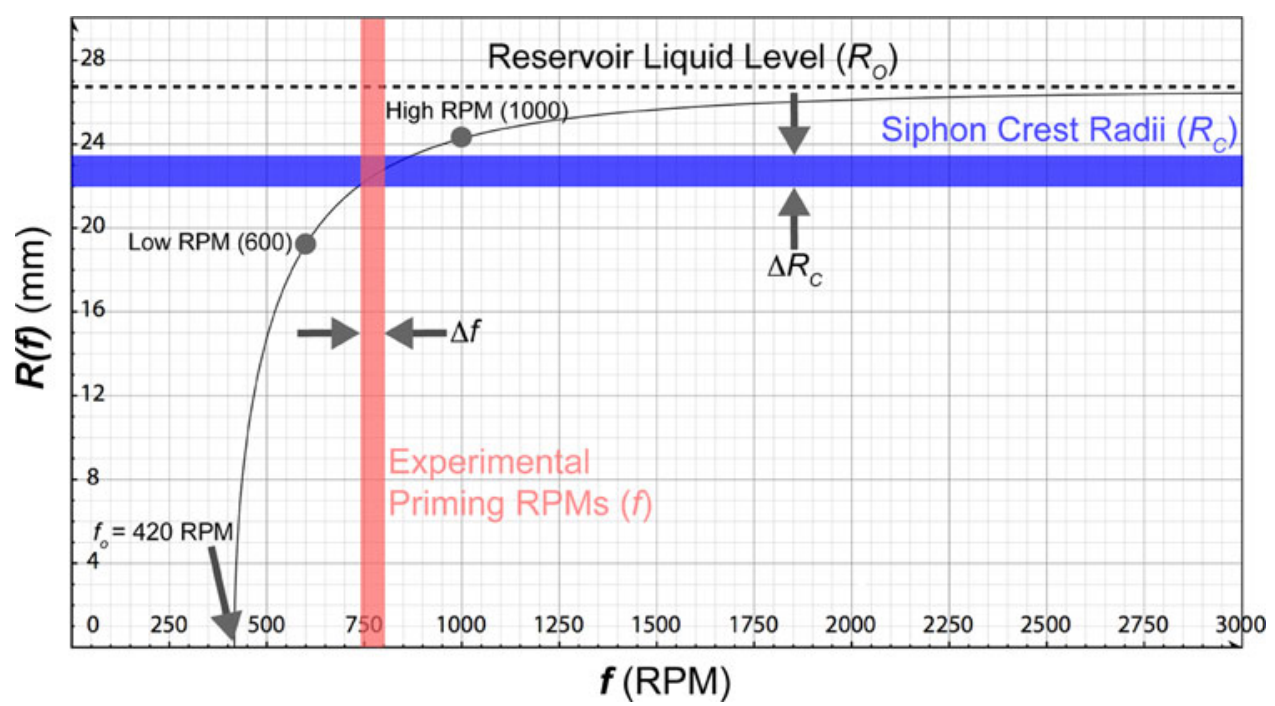

Fig. 6 The function $R(f)$ (calibration curve) as given from Eq. 3, showing the position of the crests and working frequencies (high and low) highlighted. The horizontal (blue) strip indicates the positions of the crests. The vertical (red) strip indicates the experimental RPM

\section{Results and discussion}

The triple serial siphon presented shows excellent experimental functionality at the high and low RPM values used for testing (Fig. 4). The siphons all primed as expected, and the inline capillary valves prevented the siphons from priming more than one at a time. As can be seen in Fig. 4, the cyclic high and low spin speeds provided the required functionality to subsequently overcome the inline capillary valves and allow the siphons to prime.

The analytical analysis performed provides insight into the characteristics of serial siphon valves as a practical guide for serial siphon design. Namely, a function, Eq. 3, was determined to calculate the critical siphon priming frequency, $f$, as a function of common fluidic properties (density and surface tension), surface properties (contact angle), and known microchannel geometries (chamber and siphon depths, and chamber and siphon-crest distances from the CD center). To the author's knowledge, this is the first time an analytical discussion of siphoning on a microfluidic centrifugal platform has been discussed.

As shown in Fig. 6, the observed experimental siphon priming burst frequency was $770 \pm 40 \mathrm{RPM}$, showing very good correlation with the analytical analysis which calculated, from Eq. 3, the expected average burst frequency to be 783 RPM. This represents a variation of less than $2 \%$ between the average experimental and analytical values. As the siphon priming frequency, $f$, is one of the most important practical aspects of the serial siphon valve, it has been shown that Eq. 3 provides an adequate model for this parameter. It is worth re-iterating that the model developed values observed for siphon priming. An advancing contact angle of $59^{\circ}$ is considered in both figures. Excellent correlation is shown between the experimental (vertical red bar) and analytical (overlap of horizontal blue bar and plotted line) frequency values

is valid only for siphons where the channel depth is much smaller than the width, as in the system presented. In systems where these two dimensions are similar in scale, a more complex 3-D model may be required. Additionally, the $R_{0}$ value was taken as a constant, and in systems where the liquid volume in the reservoir is not much larger than the volume contained within the siphons, changes in $R_{0}$ must be taken into consideration.

An important aspect and feature of the serial siphon is the relative lack of sensitivity to the specific high and low RPM values used. As long as the high spin speed value is above the critical priming RPM, and the low spin speed value is below the critical priming RPM and the burst frequency of the inline capillary valve, the serial siphon will function. Moreover, the specific high and low RPM values used can be drastically offset from the critical priming frequency, allowing for large variations in the spin profile. For example, in the triple serial siphon device presented, a high spin speed value of 3,000 RPM and a low spin speed value of 100 RPM could have been used. This keeps the working spin speeds far away from the critical priming frequency, allowing robust function regardless of the fluidic properties (e.g., viscosity and surface tension).

This feature of the serial siphon also provides relative insensitivity to degradation in surface treatments used for hydrophilization. For example, if the system presented here had an initial contact angle of $30^{\circ}$, Eq. 3 can be used to calculate the initial average critical priming frequency of $\sim 1,800$ RPM. If the contact angle increased to $59^{\circ}$, the priming frequency would drop to $780 \mathrm{RPM}$. By operating the CD at a high of 3,000 and a low of 100 RPM (beyond 
both critical priming frequencies), serial siphon functionality remains regardless of changes in contact angle. Thus, the serial siphon provides an incredibly robust serial valving technology for microfluidic centrifugal platforms.

Careful consideration must be given to the inline capillary valve required for the serial siphon valve. As discussed, the low spin frequency used must be kept below the burst frequency of the inline capillary valve. If a slightly higher frequency is used, the capillary valve will burst, the next siphon will prime, and the sequence will continue, causing failure of the device. In addition, the radial distance of the capillary valve must be kept at or below the height of the liquid in the reservoir. If kept above the reservoir liquid level, the capillary valve may never burst, and the siphons may not prime. This has important implications in systems where the liquid height in the reservoir changes significantly during serial siphon priming. The size and shape of the capillary valve also affects the amount of residual liquid left within the entire serial siphon structure after pumping is complete. Qualitatively, the amount of fluid left behind in the serial siphon structures presented here was little, but the majority was caught within the inline capillary valves.

The serial siphon improves on current serial valving techniques, namely capillary valving, on centrifugal microfluidic platforms. Serial capillary valves remain difficult to characterize due to their dependence in part on fluidic and surface properties, especially when implemented in hydrophilic materials where treatments can be time dependent. The serial siphon valve overcomes these limitations by providing a valving technology whose operation is insensitive to these properties. Moreover, use of serial siphon valves allows high CD spin speeds to be used, as they are not limited by burst frequency, as is the case with capillary valves. Spin speeds in excess of several thousand RPM can be used with serial siphon valve functionality retained.

Potential applications of this technology include designs for integrated in vitro diagnostic (IVD) devices. For example, when designing sample-to-answer CDs for NA diagnostics, the sequential analysis steps of NA extraction, amplification, and detection must be incorporated into a single disposable. The materials and reagents required for each step must be contained until needed, and then transferred to the chamber of interest in order to perform the next step. More specifically, in the integration of CD-based lysis (Kido et al. 2007) and polymerase chain reaction (PCR) (Jia et al. 2007) systems, PCR reagents must be held back while lysis takes place. By using serial siphon valves, PCR reagents can be withheld during a series of high-RPM operations that perform lysis. After the lysis operation is complete, the PCR reagents can be released into the next chamber for mixing with the lysed sample, in order to allow for PCR amplification. In another example, serial siphons could be used to control the sequential release of DNA sample, wash, and rinse solutions across an array of capture probes for DNA hybridization detection devices (Roy et al. 2008). Moreover, multiple washes and rinses could easily be added using the serial siphon valve. Thus, many CD-based IVD devices can be robustly enabled by the implementation of serial siphon valves.

\section{Conclusions}

Proof-of-concept for a novel centrifugal microfluidic serial valve based on repeated passive siphoning and valving has been reported. This design withholds fluids through multiple high and low speed operations and allows the fluids to be subsequently distributed to areas of interest. Serial siphon valving is simple to implement on centrifugal platforms, and overcomes previous problems of valve characterization due to time dependent surface treatments. More specifically, serial siphon valves provide more robust passive serial valving as compared to serial capillary valves.

Additionally, a detailed analytical analysis of serial siphons on the $\mathrm{CD}$ has been presented that can be used to describe and develop siphoning, single or serial, on a microfluidic centrifugal platform. Excellent correlation between the 2-D analytical model developed and the experimental devices tested was observed.

The serial siphon valve has numerous potential applications, and will be especially useful in the development of IVD systems for NA analysis. Integrated microfluidic NA diagnostic devices can be quite complex, and the availability of a simple and passive serial valve will eliminate the need for many of the more complex valving solutions. This is especially true for systems whose requirements for high-speed spin operations severely limit the use of capillary valves. The serial siphon valve is a powerful device, and will help to enable the development of integrated sample-to-answer CD-based diagnostic devices.

Acknowledgments The authors wish to thank Dr. Michel Bergeron's group at the University of Laval in Québec, Canada. The authors also thank Will Southard for assistance during CD fabrication, and Genome Québec for support in part of the presented research.

Open Access This article is distributed under the terms of the Creative Commons Attribution Noncommercial License which permits any noncommercial use, distribution, and reproduction in any medium, provided the original author(s) and source are credited.

\section{References}

Badr I, Johnson R, Madou M, Bachas L (2002) Fluorescent ionselective optode membranes incorporated onto a centrifugal microfluidics platform. Anal Chem 74:5569-5575 
Beaulieu I, Geissler M, Mauzeroll J (2009) Oxygen plasma treatment of polystyrene and Zeonor: substrates for adhesion of patterned cells. Langmuir 25:7169-7176

Beebe D, Mensing G, Walker G (2002) Physics and applications of microfluidics in biology. Annu Rev Biomed Eng 4:261-286

Bergeron M, Peytavi R, Kido H, Madou M (2008) Serial siphon valves for fluidic or microfluidic devices. Patent Application, $\mathrm{WO} / 2008 / 106782$

Brenner T, Glatzel T, Zengerle R, Ducrée J (2005) Frequencydependent transversal flow control in centrifugal microfluidics. Lab Chip 5:146-150

Chen J, Huang P, Lin M (2008) Analysis and experiment of capillary valves for microfluidics on a rotating disk. Microfluid Nanofluid 4:427-437

Cho H, Kim H, Kang J, Kim T (2007a) How the capillary burst microvalve works. J Colloid Interface Sci 306:379-385

Cho Y, Lee J, Park J, Lee B, Lee Y, Ko C (2007b) One-step pathogen specific DNA extraction from whole blood on a centrifugal microfluidic device. Lab Chip 7:565-573

Ducrée J, Haeberle S, Lutz S, Pausch S, von Stetten F, Zengerle R (2007) The centrifugal microfluidic bio-disk platform. J Micromech Microeng 17:103-115

Duffy D, Gills H, Lin J, Sheppard N, Kellogg G (1999) Microfabricated centrifugal microfluidic systems: characterization and multiple enzymatic assays. Anal Chem 71:4669-4678

Haeberle S, Zengerle R (2007) Microfluidic platforms for lab-on-achip applications. Lab Chip 7:1094-1110

Jia G, Ma K, Kim J, Zoval J, Peytavi R, Bergeron M, Madou M (2006) Dynamic automated DNA hybridization on a CD (compact disc) fluidic platform. Sens Actuators B 114:173-181

Jia G, Siegrist J, Deng C, Zoval J, Stewart G, Peytavi R, Huletsky A, Bergeron M, Madou M (2007) A low-cost, disposable card for rapid polymerase chain reaction. Colloids Surf B Biointerfaces 58:52-60

Kido H, Micic M, Smith D, Zoval J, Norton J, Madou M (2007) A novel, compact disk-like centrifugal microfluidics system for cell lysis and sample homogenization. Colloids Surf B Biointerfaces 58:44-51

Lai S, Wang S, Luo J, Lee L, Yang S, Madou M (2004) Design of a compact disk-like microfluidic platform for enzyme-linked immunosorbent assay. Anal Chem 76:1832-1837

Larsson A, Dérand H (2002) Stability of polycarbonate and polystyrene surfaces after hydrophilization with high intensity oxygen RF plasma. J Colloid Interface Sci 246:214-221

Madou M (2002) Fundamentals of microfabrication, 2nd edn. CRC Press, Boca Raton

Madou M, Zoval J, Jia G, Kido H, Kim J, Kim N (2006) Lab on a CD. Annu Rev Biomed Eng 8:601-628

Peytavi R, Raymond F, Gagné D, Picard F, Jia G, Zoval J, Madou M, Boissinot K, Boissinot M, Bissonnette L, Ouellette M, Bergeron M (2005) Microfluidic device for rapid ( $<15 \mathrm{~min}$ ) automated microarray hybridization. Clin Chem 51:1836-1844

Roy E, Siegrist J, Peytavi R, Diaz-Quijada G, Roberge H, Normandin F, Jia G, Zoval J, Madou M, Bergeron M, Dumoulin M, Veres T (2008) Thermoplastic elastomers (TPE) block copolymers, a new material platform for microfluidics: proof-of-concept for complex siphon valving on a CD. In: Proceedings of MicroTAS 2008 Conference, San Diego, CA

Steigert J, Brenner T, Grumann M, Riegger L, Lutz S, Zengerle R, Ducrée J (2007) Integrated siphon-based metering and sedimentation of whole blood on a hydrophilic lab-on-a-disk. Biomed Microdevices 9:675-679 